\title{
¿Cómo lograr SER UNA DE LAS MEJORES EMPRESAS PARA TRABAJAR en Colombia? El caso de Laboratorios Wyeth
}

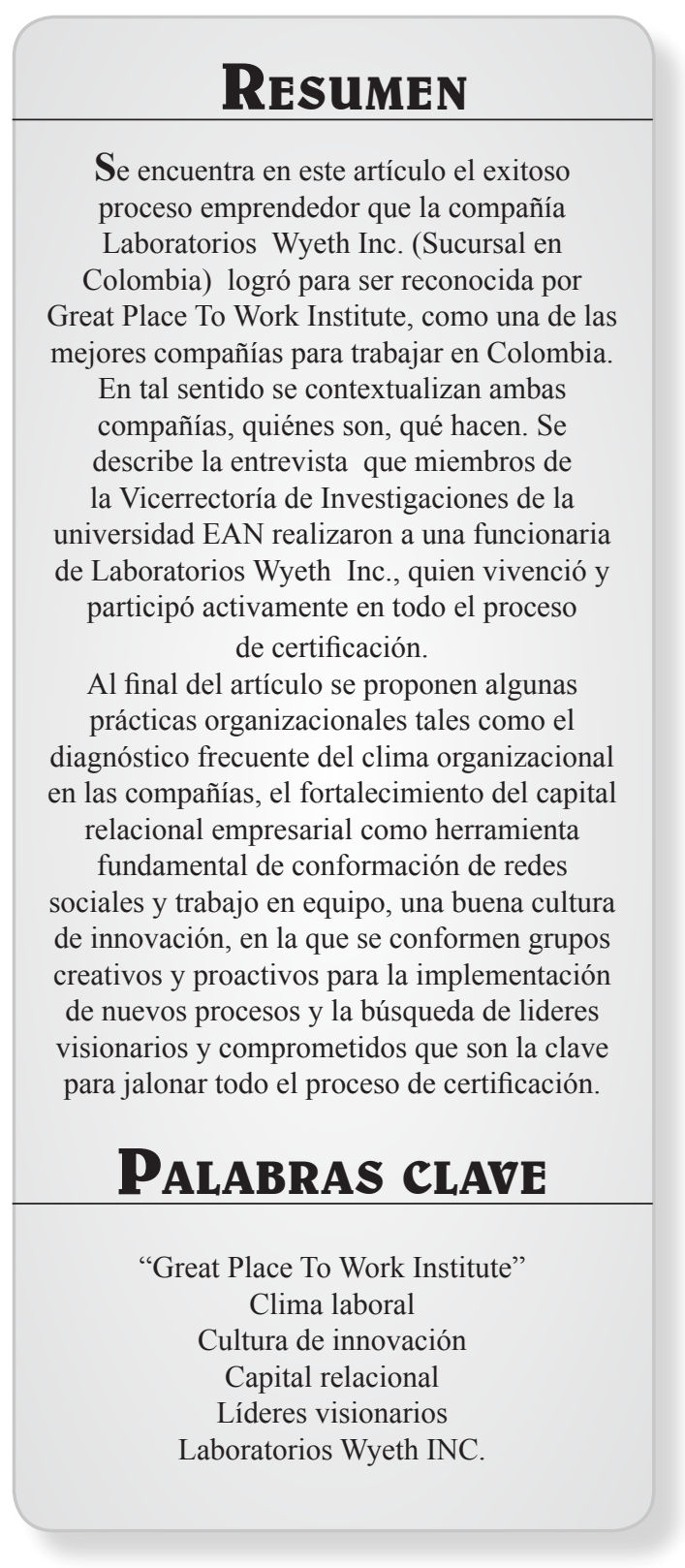

María del Pilar Ramírez Salazar*

\section{Resumen}

Se encuentra en este artículo el exitoso proceso emprendedor que la compañía Laboratorios Wyeth Inc. (Sucursal en Colombia) logró para ser reconocida por Great Place To Work Institute, como una de las jores compañías para trabajar en Colombia. sentido se contextualizan ambas de Vicerrectoría de Investigaciones de la Laboratorios Wyeth Inc., quien vivenció y de certificación.

Al final del artículo se proponen algunas prácticas organizacionales tales como el diagnóstico frecuente del clima organizacional las compañias, el fortalecimiento del capital relacional empresarial como herramienta fundamental de conformación de redes ipo, una buena cultura de innovación, en la que se conformen grupos varios y comprometidos que son la clave

\section{Palabras clave}

"Great Place To Work Institute"

Capital relacional

Líderes visionarios

Laboratorios Wyeth INC.

\section{INTRODUCCIÓN}

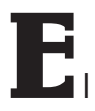

mundo empresarial se ve avocado, por el competitivo entorno económico a la permanente búsqueda de elevar sus estándares y obtener certificaciones y reconocimientos que evidencien estos logros frente a terceros. Es común escuchar en el argot empresarial que las compañías se encuentran vinculadas a nuevos procesos de certificación y estandarización de acuerdo con las necesidades de cada organización y su mercado de referencia. En Colombia la certificación más solicitada por las pequeñas y medianas empresas para el mejoramiento de su competitivad según los estudios realizados por el fomipyme año 2007 son las ISO 9001, ISO 14000, BPM (buenas

\footnotetext{
* Educadora de la Universidad Pedagógica Nacional, Administradora de Empresas de la Universidad EAN.

Especialista en Construcción de Indicadores de Gestión para la Evaluación de Instituciones de Educación Superior - Magister en Gestión de Organizaciones de la Universidad de Quebec.

Para la realización de este artículo se contó con la colaboración de Leonardo Charry, Director de Recursos Humanos; Claudia Motta, Director Asociado Entrenamiento y Desarrollo de Laboratorios Wyeth Inc. y de Rafael Pérez Uribe, Profesor Asociado de la Universidad EAN.
}

Este artículo fue entregado el 3 de mayo de 2008 y su publicación aprobada por el Comité Editorial el 18 de junio de 2008. 
prácticas de manufactura), BPA (buenas prácticas agrícolas y de almacenamiento), entre otras. Estas certificaciones son requeridas y justificadas porque aportan una mejor puntuación para la presentación de ofertas como proveedores en los procesos de licitación pública, convenios y procesos de exportaciones en la mayoría de las empresas y para acreditar ante sus stakeholders los altos niveles de calidad de los bienes y servicios, que ofrecen.

Sin embargo, estos procesos de certificación y reconocimiento están entrando en una nueva fase para potencializar al máximo todos los activos empresariales. No sólo los que se reflejan en el balance financiero de la empresa, sino a aquellos que implican una ventaja competitiva diferenciadora y permanente frente a los compertidores. Estamos hablando de elementos que en ningún caso, pueden ser imitados o copiados fácilmente y que forman parte de los denominados activos intangibles que se acumulan al interior de la organización, como resultado de su actividad diaria y de sus procesos de creación de conocimiento y es exactamente dentro de esa nueva dimensión donde nos ubicamos y detectamos el poder transformador y estratégico de gestionar el clima laboral.

Una actitud de logro, con claros retos y un entorno apropiado permiten el adecuado desarrollo del talento para la generación de mayor innovación, efectividad, confianza y probabilidades de éxito. De acuerdo con esa percepción los talentos de una organización reaccionan y se comportan como respuesta a múltiples estímulos que se identifican en el mundo del trabajo.

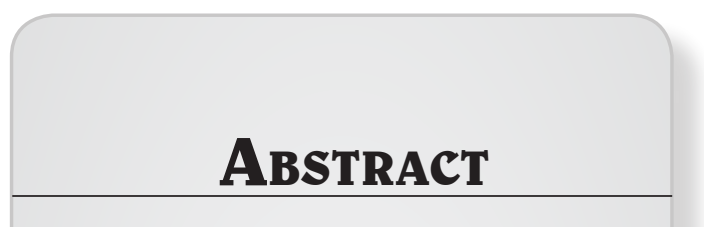

In this article, the Wyeth Inc successful entrepreneurial process is described, showing how it was certified by The Great Place to Work company, as one of the best companies to work for in Colombia.

In this way, both companies are presented, specifically who they are and what they do. The EAN University Research Unit interviewed one of Wyeth company workers who clearly experienced and participated in the certification process of this company. Finally, some good organizational practices are given, such as a frequent diagnosis on organizational climate, the strengthening of managerial relational capital as a significant tool to build up social network and team work, an efficient innovation culture in which creative and proactive groups are formed to implement new processes, and the search for visionary and compromised leaders, becoming the key to make a certification process possible.

\section{KEy WORDS}

Great place to work Work climate Innovation culture Relational capital Visionary leaders Weyth Inc Company 
Hacer una evaluación y análisis de clima laboral significa evaluar cómo los talentos perciben las problemáticas organizacionales y permiten diagnosticar una organización a través de la información recolectada para tomar acciones que generen efectos perdurables en las conductas de sus colaboradores.

Como se pone de presente, el análisis de clima laboral reviste fundamental importancia para generar un campo fértil que facilite la gestión de los activos intangibles y a la vez tomar a los demás competidores como referente y por ello nos avocamos a metodologías que nos permitan compararnos con los mejores en la categoría como son las ofrecidas por Great Place to Work Institute.

Esta forma de abordar el tema reconoce con hechos que finalmente, las personas son las que hacen la real diferencia en las organizaciones y por ello, requieren ser valoradas en su justa dimensión bajo ópticas que evalúan si los colaboradores se sienten a gusto trabajando y se identifican los niveles de confianza, orgullo y compañerismo, lo cual se comentará en este artículo.

\section{Breve reseña de la compañía Wyeth Inc. ${ }^{1}$}

Ta historia comienza en el siglo XIX por el año de 1860 cuando Frank y John Wyeth abren una farmacia en Filadelfia Pensilvania, promocionando tónicos y elixires por medio de catálogos los cuales son acogidos en Canadá y el Reino Unido. Esta firma, era más que un laboratorio. Era un centro de investigación para la elaboración de medicinas.

El éxito de esta farmacia trasciende las fronteras comenzando a expandir sus sucursales en Montreal, Canadá. Al morir John Wyeth en 1907 su único hijo Stuart hereda la firma y le deja el manejo y control de la compañía a su alma Mater, la Universidad de Harvard.
En los años de la segunda guerra mundial (1941), Wyeth fue reconocida por el gobierno americano por la contribución en medicinas y vacunas para los soldados de ese entonces. Lanza al mercado la penicilina, inicialmente se permite su fabricación para la armada nacional y luego obtiene la licencia para ofrecerla al público en general.

En 1945 adquiere la compañía Fort Dodge, de la cual se traspasó todo el conocimiento del cuidado de los animales. En los años 50 lanza al mercado medicinas reconocidas para el tratamiento del alcoholismo y en los años 60 se convirtió en líder de la vacuna contra el Polio.

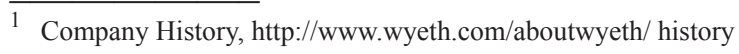


En 1993 funda el primer centro de investigación para la salud de la mujer con grandes resultados en la disminución los síntomas de la menopausia y el mejoramiento de los comportamientos generados por la depresión. Con diferentes productos entre ellos el multivitamínico Centrum.

AHP (American Home Products) como holding del grupo, interesada por focalizarse en la investigación y desarrollo de productos para el cuidado de la salud que mejoran la vida, refuerza su portafolio de productos en terapias especializadas de carácter biológico y en el año 2002 cambia su nombre por el de Wyeth que mantiene actualmente.

Logra en el año 1994 elevar sus ventas a \$13 Billones de dólares, posicionándose como una de las mejores compañías preocupadas por el mejoramiento de la salud.

En la actualidad Wyeth tiene tres unidades de negocio:

\section{- Wyeth Pharmaceuticals}

\section{- Wyeth Consumer Healthcare}

\section{- Fort Dodge Animal Health}

En Colombia, Laboratorios Wyeth Inc. inició oficialmente su operación en el año de 1952. Sin embargo, desde el año de 1939 el señor José Fulladosa, inició sus primeros contactos para importar Aldrox, Kaomagma, Lentopen (penicilina procaínica), SMA, vitaminas y laxantes a través de la firma colombiana Cabarría \& Cía Ltda. Dichos productos fueron lanzados al mercado progresivamente y vendidos por el Señor Fulladosa y tres representantes de ventas.
La producción empezó en Cali con la colaboración de Home Products. Posteriormente se trasladó a Bogotá cuando la primera planta de leches fue inaugurada en el año 1958 y la de la línea farmacéutica en 1960.

Alrededor del año 1957, los Laboratorios Ayerst, que hoy son parte integral de Wyeth, comenzaron sus ventas, con productos importados, principalmente el Ayerogen, el Halothane y el Jarabe Clusivol. Hacia el año 1975 compró el Laboratorio Nacional Hormona, que en 1943 había construido sus instalaciones en Bogotá en la Avenida Suba No. 96- 66, que en la actualidad sirven como oficinas administrativas de Laboratorios Wyeth Inc. El primer Gerente General de Hormona, el señor Esteban Rosemberg, trabajó al lado del Dr. Carlos Lleras Restrepo, que se desempeñaba como Presidente de la Junta Directiva y quien llegó a la Presidencia de la República de Colombia para el periodo 1966 a 1970.

Sus primeros productos fueron Mareol, Codeinetas y algunas hormonas femeninas. El primer presidente de Ayerst-Hormona, Sr. Fred Badía, quien dirigió la fuerza de ventas de ese entonces con 20 representantes y en 1987 Laboratorios Wyeth Inc. y Laboratorios Ayerst-Hormona S.A. se fusionaron y se designó al Doctor Ernst Adler como Gerente General de ambos laboratorios hasta el año 1999.

En Agosto de 1999 se nombró al Doctor Carlos Alberto Serrano en la posición de Gerente General como parte del proceso de modernización de las operaciones en el país y llegar así a ofrecer a la comunidad un conjunto de productos de las más altas calidades técnicas en apoyo a las labores de salud pública en la nación. 
La siguiente tabla muestra las ganancias de Wyeth en el primer trimestre del año 2008, en sus tres grandes divisiones a nivel mundial.

CUADRO No. 1

Utilidades totales netas por segmento

Primer trimestre (Marzo 31 de 2008)

\begin{tabular}{|l|c|c|}
\hline $\begin{array}{c}\text { GANANCIA NETAS REPORTADAS } \\
\text { POR SEGMENTO }\end{array}$ & MILLONES DE US\$ & INCREMENTO \\
\hline FARMA & 4,759 & $6 \%$ \\
\hline Consumer Healthcare & 675 & $10 \%$ \\
\hline Animal Health & 277 & $0 \%$ \\
\hline CONSOLIDADO TOTAL & 5,711 & $6 \%$ \\
\hline
\end{tabular}

FUENTE. http://www.wyeth.com/news?nav=display\&navTo=/wyeth_html/home/news/announcements/2008/ 1216810804325.html (Adaptado por la autora de este artículo).

Algunos de los principales logros, descubrimientos e innovaciones farmacéuticas acontecidos a lo largo de la historia de Wyeth, han sido:

- Desarrollo de los comprimidos y la primera máquina de comprimir rotativa.

- Primeras cápsulas de gelatina soluble.

- Primer estrógeno oral activo.

- Primera presentación oral de una vacuna trivalente para la polio en EE.UU.

- Desarrollo de la vacuna que ayudó a erradicar la viruela en todo el mundo.

- Primera síntesis de esteroides para la contracepción oral.

- Primera vacuna para la difteria / tétanos / pertusis acelular disponible en EE.UU.

- Primera vacuna conjugada de Haemo-philus B para uso en niños.

- Primera vacuna conjugada contra Meningococo C para uso en niños y adolescentes.

- Única vacuna conjugada contra Pneumococo.

- Primer inhibidor de la recaptación de Serotonina y Noradrenalina para el tratamiento eficaz de la depresión y la ansiedad generalizada.

- Primera combinación de estrógenos y progestágenos en un solo comprimido para la terapia hormonal sustitutiva.

- Único factor IX recombinante para la hemofilia B y el primer factor VIII recombinante de $2^{\mathrm{a}}$ generación para la hemofilia A. 


\section{Algunos reconocimientos y premios A NIVEL MUNDIAL ${ }^{2}$}

- En el 2007, La revista ' $R \& D$ Directions' comunicó en el ejemplar de enero que seleccionó a Wyeth Pharmaceuticals "El mejor pipeline en Sistema Nervioso Central". Actualmente Wyeth tiene 19 componentes en su línea de fármacos del Sistema Nervioso Central bajo desarrollo como tratamientos potenciales para pacientes con condiciones neurológicas patológicas, incluyendo esquizofrenia, trastorno bipolar, depresión, enfermedad de Alzheimer, dolor neuropático, fibromialgia y derrame cerebral grave.

- En eL 2006, en el mes de noviembre, la revista 'Scrip World Pharmaceutical News' concedió el premio al "Mejor equipo directivo del año" al comité ejecutivo de Wyeth I+D, por ser "mucho más que la suma de sus partes". Este equipo, que puso en funcionamiento hace seis años un nuevo modelo productivo, dirige a más de 7.000 investigadores en todo el mundo.
- En el 2005, en la ceremonia inaugural de los Scrip Awards - celebrada en Londres en diciembre de 2005 - Wyeth recibió dos galardones de la Industria Global: "Best Management Team" y "Best Partnership Alliance".

"The Discoverers Award", el premio científico de mayor relevancia en la industria farmacéutica de los EE.UU. fue concedido a los científicos de Wyeth Research: Dr. Ronald J. Eby, Dr. Dace Madore y Dr. Velupillai Puvanesarajah.

- En el 2004, Wyeth recibió, por tercera vez en cuatro años (2000, 2002 y 2003), el premio concedido por AIMFA (Agrupación de Investigación y Marketing Farmacéutico) y patrocinado por IMS Health, a la compañía con el más alto crecimiento en ventas en España durante el año 2003.

En Suramérica, Wyeth tiene sucursales en Argentina, Chile, Ecuador, Venezuela, Perú y Colombia.

\footnotetext{
2 http://www.wyeth.es/Inicio/ConoceWyeth/Premiosyreconocimientos/tabid/187/Default.aspx, extractado el 28 de mayo de 2008 de la página principal de Laboratorios Wyeth.
} 


\section{Cultura Wyeth}

17 yeth construye una cultura organizacional que refleja el compromiso con su gente, la preocupación constante de mejorar la calidad de vida de sus colaboradores y llevar a cabo planes y programas que coadyuven al logro de los objetivos estratégicos de la empresa.

Según lo manifiesta el Director Regional de Recursos Humanos de Wyeth, Leonardo Charry, en la compañía se ha tenido en cuenta el enfoque del Balanced Scorecard de Kaplan y Norton ${ }^{3}$, quienes comenzaron a medir los bienes intangibles de la empresa, para soportar el crecimiento y desarrollo sistémico.

Wyeth tiene en cuenta la perspectiva de sus clientes y pacientes, preocupándose por conocer sus necesidades y prioridades, de tal forma que se logre ofrecer los productos más adecuados para las distintas poblaciones, con tecnología de punta, velando por la calidad de todos los procesos de la compañía y fomentando el aprendizaje y desarrollo de conocimiento de todos sus colaboradores, mediante la implementación de modelos de competencias, gestión del conocimiento e información que facilitan y mejoran la eficiencia de sus tareas diarias.

Esta cultura de reconocimiento al valor del capital intelectual $^{4}$ de sus colaboradores como base esencial de la productividad y sostenibidad de la empresa, motivó a sus directivos a la búsqueda de una mejora continua en su clima laboral y buscar su reconocimiento externo.

\section{4. ¿Por qué Wyeth busca a Great Place to Work INSTITUTE PARA QUE LE ASESORE EN EL PROCESO DE CONVERTIRSE EN UNO DE LOS MEJORES LUGARES PARA TRABAJAR EN Colombia?}

El equipo directivo de Wyeth conciente del impacto del clima organizacional en la vida de la empresa y en la generación de sus resultados, se comprometió con la medición del clima laboral y con el compromiso de implementar un plan de acción con base en los resultados que se obtuvieran.
Se revisaron diferentes metodologías y se escogió la diseñada por Great Place To Work Institute (GPTW) para efectuar el diagnóstico, identificar oportunidades y fortalezas junto con la decisión de iniciar una metodología de intervención en los procesos críticos de la gestión estratégica, gestión de personas y

\footnotetext{
${ }^{3}$ Balanced Business Scorecard (Kaplan Y Norton, 1996).

${ }^{4}$ Euroforum (1998), el Capital Intelectual se compone de, Capital humano, estructural y relacional.
} 
mejora en los procesos de comunicaciones internas y obtener así en un corto periodo, una mejora sustancial del clima y ser reconocida como una de las mejores empresas para trabajar en Colombia.

Los fundadores de GPTW Institute, Amy Lyman y Robert Levering, han puntualizado su aporte en generar una cultura basada en dimensiones de liderazgo que impactan el clima laboral en las organizaciones y por ende, la productividad de las mismas. La Doctora Amy Lyman, es cofundadora, y actualmente Presidenta del Consejo de Directores así como Presidenta del Comité de Estrategia de Great Place to Work Institute, quien además de supervisar la Investigación y el análisis en el Instituto, trabaja con líderes y directivos en la interpretación de resultados y el diseño de planes de acción, apoyándoles en los procesos de transformación de sus organizaciones para ser excelentes lugares para trabajar. Desarrolla su trabajo a nivel individual y organizacional para lograr que los objetivos deseados sean sostenibles a largo plazo. Robert Levering, es coautor de la lista anual de la revista Fortune "100 Best Companies to work to". Es un estudioso del fenómeno de excelentes lugares para trabajar durante más de veinte años. Junto con el coautor Milton Moskowitz, escribió el primer best-seller sobre este tema en 1984, "The 100 Best Companies to Work for in America"5(cuatro años después, publicó "A Great Place to Work: What makes some employers so good - and most so bad", un completo estudio sobre los excelentes lugares para trabajar. Ha escrito seis libros más y decenas de artículos en muchas publicaciones internacionales, incluido el Financial Times. En 1990 fundó (con Amy Lyman) el Great Place to Work $\circledR^{\text {Institute. }}$

Tanto Levering como Lyman consideran que uno de los principales factores que influyen en reconocer a una empresa como mejor lugar de trabajo es la confianza, que la describen bajo las tres premisas siguientes:

1. Credibilidad en la administración: que tanto los empleados creen lo que sus líderes les dicen.

2. Respeto hacia los empleados: demostrado en el interés de los administradores en el desarrollo profesional y personal de sus empleados. Qué tanto muestran aprecio por su trabajo y se preocupan por ellos como personas.

3. Un trato justo: cómo las personas son consideradas en términos de salarios, promociones y reconocimiento.

\footnotetext{
5 @ 2008 Cable News Network., http://money.cnn.com/magazines/fortune/bestcompanies/2008/full_list/ A
} 


\section{Qué valora Great Place to Work Intitute}

1 odas las empresas que se inscriben para el proceso son evaluadas bajo dos instrumentos estándar: "Trust Index" y el "Culture Audit". En esos instrumentos recogen toda la información a ser analizada, tabulada por profesionales que guardan absoluta confidencialidad de los datos.

El Great Place to Work $®$ Trust Index $@$ es una encuesta de plantilla que identifica los niveles de confianza, orgullo y compañerismo dentro de su entorno de trabajo. La opinión de los empleados en la encuesta tiene la mayor importancia para la evaluación final la cual definirá los puestos en las listas de "Best Workplaces". Todos los participantes realizan la encuesta.

La siguiente información explica más al fondo el contenido de la encuesta "The Trust Index@:

- Consta de 55 preguntas sobre credibilidad, respeto, trato justo, orgullo y compañerismo - las cinco dimensiones que corresponden al Great Place to Work® Mode/@.

- Las preguntas son positivamente redactadas y provienen de las cinco dimensiones del Modelo. Los siguientes son ejemplos de preguntas:

- Credibilidad: la dirección me mantiene informado/a sobre las cuestiones y cambios importantes. La
Dirección da mucha responsabilidad a los trabajadores.

- Respeto: la dirección implica a la plantilla en la toma de decisiones que afectan a su trabajo y a su entorno laboral. Se me ofrece formación y desarrollo para poder avanzar profesionalmente.

- Contiene una pregunta a desarrollar, que permite a los empleados, con sus propias palabras, describir lo que sea único o inusual de su entorno de trabajo.

- Demora solamente 15 minutos para diligenciar.

Great Place to Work $®$ Culture Audit@ es una encuesta para el departamento de recursos humanos, que se utiliza para obtener un mayor conocimiento de la cultura de la organización. La encuesta está solamente disponible para participantes de las listas. Sin embargo, la siguiente información ofrece más detalles del concepto de la encuesta.

Esta encuesta tiene dos partes. La primera trata de datos demográficos de la plantilla (por ejemplo número de empleados en el país, rotación voluntaria, edad y antigüedad de los empleados, etc). También se solicita información general sobre la organización (por ejemplo año de fundación e ingresos), beneficios adicionales (por ejemplo centro de gimnasio en la compañía, seguro pagado por la organización, días de vacaciones, etc). 
La segunda, parte de la encuesta Culture AuditC contiene varias preguntas a desarrollar, que dan a la organización la oportunidad de elaborar diferentes aspectos de la cultura.
Las preguntas en la segunda parte están relacionadas con el Great Place to Work ${ }^{\circledR}$ Mode/@, que es la base de la evaluación.

\section{El proceso GPTW en Laboratorios WyETH INc ${ }^{6}$}

\section{$\mathbf{L}$} razón por la cual se escogió el enfoque de GPTW, es porque está alineado conceptualmente con el desarrollo de las variables incluidas en la dimensión de aprendizaje del modelo de mapa estratégico implementado en la empresa con fundamento en los desarrollos conceptuales que sobre el tema han desarrollado Kapplan y Norton y que se apalancan perfectamente con el tema de clima laboral y las cinco dimensiones que se revisan. De hecho son la clave para medir el clima laboral en la compañía y se vienen trabajando de tiempo atrás en la empresa.

Este proceso inició hace tres años de una forma estándar en Colombia. Se tuvo un acercamiento con la seccional del Instituto GPTW, en Bogotá, Colombia, para entender en detalle cómo funcionaba e inmediatamente se inició en la empresa con esta metodología.

Realizan la medición una vez al año y la preparación que se debe hacer antes de la implementación. Se estructura un cronograma para tener muy claro cuáles son las fechas de la medición. La primera prueba se hace en octubre de cada año. Es una encuesta estándar donde se miden cinco dimensiones: credibilidad, respeto, orgullo, imparcialidad y camaradería.

Cada una de ellas tiene dentro de sí varias variables. En credibilidad, por ejemplo, está el tema de comunicación, de difusión de objetivos y cada una tiene 6 comportamientos identificables.

Los 240 empleados de Wyeth, han participado en las encuestas y las respuestas son confidenciales. GPTW Institute envía directamente un correo electrónico con el link a cada uno de los trabajadores para recibir la información y diligenciar la encuesta. Es importante que los empleados sepan que lo que ellos manifiestan es totalmente confidencial.

El $100 \%$ de la compañía responde la encuesta, que se devuelve automáticamente al instituto GPTW. Ellos reciben todos los resultados, los procesan, hacen los análisis y generan el informe final con recomendación y con base en el se inicia el proceso de ubicar

\footnotetext{
${ }^{6}$ Entrevista realizada en Bogotá, en las instalaciones de Laboratorios Wyeth Inc. a la señora Claudia Motta y al señor Leonardo Charry, por María del Pilar Ramirez y Rafael Perez Uribe, en el 2008.
} 
a cada compañía en el ranking general. Cada empresa recibe los resultados por áreas específicas, tomando como referencia el mercadeo colombiano y el del grupo de empresas del sector económico en que se encuentra Laboratorios Wyeth Inc.

Ahora bien, en forma más detallada, podemos comentar que Great Place to Work Institute hace el análisis y presenta los resultados en términos de porcentaje de aceptación de cero a cien. Por ejemplo, en la variable credibilidad -información- comunicación -en Laboratorios Wyeth se obtuvo un puntaje de $80 \%$, que es el porcentaje de aceptación, es decir, que el $80 \%$ de las personas estuvieron muy de acuerdo con que la variable comunicación estaba en el nivel adecuado.

En el informe compara a la compañía con las diez mejores del mercado. Por ejemplo, en la variable comunicación muestran que Wyeth presenta el $80 \%$ y que las diez mejores del mercado tuvieron $90 \%$. Ese es el máximo nivel de detalle que se muestra.

En esta prueba se hace una pregunta final que llaman "el gestalt", que es un indicador que independientemente de las variables que están en la encuesta, pregunta por aparte "¿Usted considera que Wyeth es un gran lugar para trabajar? Esa es la última de las mediciones que hacen y es uno de los elementos más fuertes que utilizan para la comparación de la empresa.

Cada área hace su análisis específico de cuáles son los elementos por mejorar y los elementos altos que se pueden mantener. Sobre estos resultados, cada líder diseña su plan de acción y es el responsable por su implementación.
Hay una segunda revisión general de la compañía liderada por la Dirección de Recursos Humanos. Se analizan cuáles son los elementos bajos y que se quieren mantener comunes para toda la organización. Con esta información, se diseña un plan general para manejar los elementos que como compañía presentan mayor oportunidad de evolución. De esta manera, se recogen las dos dimensiones: a nivel general de la compañía que Wyeth ha denominado plan sombrilla y a nivel específico de cada una de las áreas que deben implementar cada uno de los líderes con sus equipos.

Los ejecutivos de recursos humanos son los facilitadores de este proceso, tanto en el diseño de los planes de acción como en su implementación y seguimiento. Un elemento importante para mejorar el clima es crear la conciencia, que el responsable del clima no es el área de Recursos Humanos, sino que es cada uno de los líderes con gente a cargo. El área de Recursos Humanos facilita y provee herramientas.

En el primer año Wyeth Colombia fue ubicado en la posición 25 del ranking de las mejores empresas para trabajar en el país. GPTW Institute presentó los resultados y se encontraron algunas claras oportunidades para trabajary mejorar en términos de factores que impactan favorablemente el clima laboral, tanto a nivel general de la compañía como a nivel del interior de las distintas áreas. Fue la primera vez que la empresa tenía una foto en términos cuantitativos de su clima laboral y se definieron las variables a trabajar durante el año siguiente. 
En la evaluación del siguiente año de las mejores empresas para trabajar en Colombia, Wyeth Colombia se ubica en la posición 16 del ranking, reflejando de esta manera la percepción de los colaboradores frente al mejoramiento del clima laboral de la organización.

\section{Un PLAN DE acción alineado a LA Evaluación DE GPTW INSTITUTE}

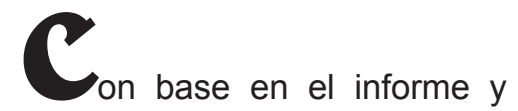
análisis de GPTW Institute se definió a nivel del comité de dirección de Wyeth Colombia, un plan de acción anual para mejorar la situación de las distintas variables que la evaluación detectó con mayores oportunidades, como se indica a continuación.

\section{Clara visión de futuro}

Con el propósito de alinear todos los esfuerzos en la organización se ha venido manejando un modelo estratégico de negocios en el cual tienen la posibilidad de participar todas las personas que ocupan los distintos niveles organizacionales. Lo que permite definir claramente el aporte esperado de cada posición en la organización fundamentados en el conocimiento de las estrategias de negocio, tácticas, metas y planes de acción de todas las áreas de la empresa. Los colaboradores saben para dónde va la organización y como su aporte es valorado.

\section{El bienestar comienza por casa}

Como actividades de apoyo Wyeth Colombia ha venido invirtiendo en programas de bienestar para los colaboradores y en algunos casos ha incluido a las familias de estos. Se analizó que el elemento de balance de la vida personal versus la laboral tenía mucha oportunidad de mejora y se diseñó e implementó el programa VISA (vida saludable), que consiste en una serie de actividades durante el año en donde cada una de las personas escogía cuál actividad extralaboral desea practicar, de forma tal, que pudiera balancear un poco su día a día, su cotidianidad, que no fuera solamente ir de la casa al trabajo y del trabajo a la casa, sino que pudiera también aprovechar sus habilidades, su talento y sus intereses en otras cosas.

A principios del año se preparó una completa programación con actividades deportivas y artísticas para todos los colaboradores (se ofreció yoga, aeróbicos, natación, cocina, pintura, etc,). Una cantidad de actividades que les permitía a las personas cambiar de ambiente y relacionarse con otras personas pero a nivel diferente de trabajo, reconocer sus habilidades en un plano diferente al trabajo y manejar el estrés laboral.

A los colaboradores se les sorprende con nuevas actividades para mantener su atención. Por eso para el 2008, se está desarrollando otro programa de bienestar que los sorprenderá y atraerá mejorando su participación, motivación y compromiso. 


\section{Imprimiendo permanente inno- vación a los programas}

Con todos los actores, tanto los líderes de los procesos como los empleados de la compañía, la innovación se construye por medio de encuestas y reuniones focales.

Antes de desarrollar el programa VISA se realizó una encuesta a una muestra de los empleados, en donde se preguntó qué entendían ellos por balance de día personal laboral. Porque para cada persona hay una connotación diferente. Esta encuesta permitió conocer los intereses de los colaboradores, sus expectativas y sus deseos. De tal forma que se diseñó este programa de acuerdo con sus propias necesidades.

En el primer año de desarrollo se realizó otra encuesta para evaluar la sostenibilidad del programa y entender cómo estaban viendo el programa VISA y como resultado se analizó que el servicio de este programa ya estaba desgastado y que se debía innovar con otras iniciativas.

En el segundo año el haber mejorado de posición indica que todos los programas y mejoras que se hicieron en la compañía fueron significativos para los empleados de Wyeth.

\section{Reforzar la estrategia de comunicación interna}

Con el propósito de mejorar a todos los niveles la comunicación se crearon foros para divulgar resultados, programas e iniciativas, junto con herramientas informativas como boletines, mensajes por correo electrónico, mensajes dinámicos en pantallas de plasmas y rodear todas las actividades de los colaboradores con un apoyo permanente de mercadeo corporativo interno.

\section{Impacto que tiene este pro- grama en relación con la productividad}

Se han realizado varios estudios donde se ha identificado la correlación a nivel general en la mejora del clima laboral con el resultado de las empresas. Un hecho en donde se demuestra que el valor de las empresas versus un clima laboral alto implica un mayor valor en el mercado. Si no se creyera en este aspecto y si no fuera importante no se realizaría. Los resultados que se ha tenido en términos de negocio han sido unos de los mejores comparándolos con el 2007.

Tanto el índice de clima y los resultados de negocio, han venido mejorando con el tiempo.

\section{Compromiso de los líderes de los procesos en el programa}

Se definen unos objetivos de crecimiento por área, lo cual es un motivador grande. Se entregan a cada área los resultados de crecimiento y lo que se espera de ellos para el próximo año.

Estos objetivos los provee el instituto Great Place To Work, para cada área específica. Por ejemplo, con el área de finanzas uno de los objetivos de crecimiento es la variable de comunicación que estaba en $\mathrm{x} \%$ y se 
espera que para el próximo año suba a $\mathrm{x} \%$. El hecho de que cada área tenga objetivos claros cuantitativos de crecimiento hace que cada líder se sienta dueño del proceso y tenga un elemento de medición claro.

Recursos Humanos acompaña a los líderes en la definición de sus planes de acción a principio de año. Después de plantear los objetivos que se quieren, se elaboran los objetivos específicos. Hay una reunión conjunta con los líderes de cada una de las áreas para identificar las medidas de acción y los indicadores.

\section{Aspectos claves que hacen que Wyeth sea un gran lugar para trabajar}

- La dinámica y el reconocimiento que tienen los líderes con cada uno de sus grupos. Los líderes realmente tienen como prioridad la comunicación con sus colaboradores. Este año se entregó un KIT de reconocimiento a cada líder que contenía diferentes tarjetas corporativas con logo Wyeth. Unas de buen trabajo, otras de gracias, de felicitaciones, etc. Este reconocimiento informal es un factor motivacional diario para los colaboradores.

- El reconocimiento por resultados esperados: también hay reconocimientos formales como viajes, bonificaciones, comisiones, encuentros, eventos, a los funcionarios que cumplen las metas o las sobrepasan.
- Definición clarísima de los objetivos: el hecho de que el personal tenga claro hacia dónde va y qué tiene qué hacer y cómo se ligan esos objetivos específicos con los objetivos generales de la compañía hacen que el personal de Wyeth esté convencido de que se convierta en un gran lugar para trabajar.

- El elemento de desarrollo del talento humano es clave sobre todo en las áreas comerciales en donde cada representante y cada ejecutivo tiene un plan individual.

- La comunicación interna de la empresa. Toda la compañía está enterada en todo momento, de los resultados de cada área, de qué está pasando en el momento, de prioridades etc. El gerente de comunicaciones utiliza diferentes medios como la intranet, pantallas de plasma, reuniones regionales etc.

- Facilidad de llegada a la oficina por el Pico y placa*: se otorga la facilidad de horarios a las personas para el acceso al trabajo. Pueden llegar después de las 9:30 cuando su carro tiene pico y placa.

- Las actividades de Bienestar: los programas de beneficios para empleados y para sus familias hacen de Wyeth una gran familia en donde se involucra también a sus mascotas.

\footnotetext{
* El pico y placa en Colombia se refiere a una restricción a la circulación vehicular por días, teniendo en cuenta el último dígito de la placa del carro.
} 


\section{Dificultades presentadas en este proceso}

Se han efectuado pocas mediciones intermedias de los programas que se van adelantando. Por ello, no se tienen indicadores de evolución a través del año que midan el impacto en cortos periodos de los esfuerzos y sólo se evalúan al final de cada año, cuando ya es poco lo que se puede cambiar. Se hace necesario implementar un programa estructurado de monitoreo de las iniciativas y acciones para poder redefinir algunos programas a tiempo y garantizar los resulta-dos en todas las intervenciones.

\section{Buenas prácticas SUGerida para las empresas QUE DEBEN INICIAR CON EL PROCESO DE CERTIFICACIÓN de “Great place to work"}

- Diagnóstico inicial del clima organizacional.En el momento en que una empresa toma la decisión de entrar al proceso de la certificación es aconsejable analizar su actual clima organizacional. Se debe realizar un diagnóstico cualitativo, que por medio de reuniones con la metodología de focus group, permitan como plantean Bolman \& Deal 1984, conocer aquellas fuerzas internas de poder, que se viven al interior de la empresa, identificar los líderes innatos, los colaboradores apáticos, el perfil de los jefes actuales, los grupos de poder y concentración de roscas etc. Estos referentes le servirán a la alta gerencia para poder reunir a sus ejecutivos y comprometerlos en el proceso a iniciar esperando de ellos que se conviertan en líderes visionarios y dinámicos para la empresa.

- Conformación de líderes dinámicos y visionarios. Es necesario como lo explicaba la Dra. Motta en este artículo que exista compromiso por parte de los líderes para hacer del proceso de certificación un éxito. Todos los líderes deben comprometerse al $100 \%$ con la convicción que la mejora del clima laboral beneficiará el desempeño de su unidad. Los líderes deben ser dinámicos, visionarios como señala Pérez 2007, deben procurar mejorar el trabajo en equipo, las relaciones interpersonales, propiciar espacios de confianza, de respeto, de reconocimiento, de camaradería de tal forma que se respire una cultura rafting en su equipo de trabajo.

La inteligencia emocional (Howard Gardner, 1983) juega un papel crítico en la conformación de los líderes dinámicos y visionarios. Un buen líder debe ser capaz de entender a sus colaboradores como lo plantea Goleman1998, las personas deben tener capacidad de reconocer sus propios sentimientos y los de los demás, de aprender a automotivarse y controlar las emociones. 
- Crecimientodecapital relacionalesencial. Indudablemente la relación que existirá entre los colaboradores de la empresa debe pasar de una relación idiosincrática (Ordoñez de Pablos, 2003) a una relación de carácter esencial, es decir las conversaciones, reuniones, y demás relaciones que se generen al interior y exterior de la empresa, deben propender a la obtención de un resultado y valor agregado esperado. Reunirse por reunirse sin objetivos claros es un desgaste de tiempo y desmotiva al personal de una empresa. Las relaciones entonces, se deben organizar bajo un esquema de alcance de metas específicas. En el proceso de mejora de un clima organizacional se puede caer en el error de creer que es suficiente con diseñar por ejemplo un programa de tertulias sociales todos los viernes. Sin embargo, el primer viernes puede ser motivador para los colaboradores pero los siguientes, pueden ser un martirio. Hay que tener en cuenta que el aumento de capital relacional no se refiere solamente a hacer amigos, es una herramienta esencial y multiplicadora para generación de conocimiento.

- Cultura de innovación. Es la época de sorprender a los clientes con servicios que ellos no lo esperan y que los hacen tener una mayor fidelidad y sentido de pertenencia hacia una empresa. En ese sentido la compañía debe crear un equipo dinámico, emprendedor, innovador que sea capaz de diseñar programas para los empleados que aumenten su compromiso y motivación al trabajo. En la literatura se encuentran autores como (Nonaka Ikujira, 2000) en donde la clave de la innovación y de la creación de valores agregados en la empresa permitan a la empresa ser productiva, competente y eficiente. Para Nonaka, la imaginación, la creatividad, la inventiva, y la capacidad de soñar, son aspectos claves en el desarrollo de productos y servicios que conquisten mercados.

Se encuentran consejos prácticos del éxito de la Pymes que innovan en el artículo escrito por (Castellanos Gonzalo, 2003), que manifiesta que la innovación exige nuevas formas de relación entre las personas, nuevas formas de organización, de colaboración y de aproximación. Implica además creación de ambientes de confianza, de apadrinamientos para favorecer la experimentación con su secuela de éxitos y fracasos. Según lo manifiesta (Blanco Carlos, 2008), en su boletín $\mathrm{N}^{\circ} 8$ de Documentos deAdministración, la universidad cumple un papel fundamental en el siglo XXI. Los futuros profesionales tendrán el reto diario de la capacitación del nuevo conocimiento, serán quienes lideren procesos que apunten a la ventaja competitiva sostenible de las empresas por el valor añadido e innovación que aportan. Para la cultura de la innovación, la empresa debe encontrarse en el estadio proactivo (Mendoza, José María, 1996), que exige de los pronosticadores su más alto desempeño para prever las condiciones en el futuro y así poder adaptarse, anticipándose a las circunstancias y detectando oportunidades de negocio de forma temprana, con el fin de tener una alta ventaja competitiva. Los creadores e innovadores del sistema de aprendizaje actúan decididamente y se revalúan permanentemente. 
ANEXO

LAS MEJORES EMPRESAS PARA TRABAJAR EN COLOMBIA 2007 SEGÚN GREAT PLACE TO WORK

(La lista de las mejores empresas para trabajar en Colombia 2007 fue generada por Great Place to Work ${ }^{\circledR}$ Institute Colombia y publicada por la Revista Dinero)

\begin{tabular}{|c|c|}
\hline RANGO & COMPAÑÍAS \\
\hline 1 & Telefónica Móviles de Colombia S.A. \\
\hline 2 & Suramericana de Seguros S.A. \\
\hline 3 & Quala S.A. \\
\hline 4 & Colombiana Kimberly Colpapel S.A. \\
\hline 5 & C.I. Wayuu Flowers S.A. \\
\hline 6 & Productos Roche S.A. \\
\hline 7 & Suratep S.A. \\
\hline 8 & Construcciones el Cóndor S.A. \\
\hline 9 & McDonald's Colombia \\
\hline 10 & Yanbal de Colombia S.A. \\
\hline 11 & Petrobras Colombia Ltda. \\
\hline 12 & $\begin{array}{l}\text { Dow Química de Colombia S.A. y Dow AgroSciences } \\
\text { de Colombia S.A. }\end{array}$ \\
\hline 13 & Belcorp \\
\hline 14 & Codensa S.A. \\
\hline 15 & Nestlé de Colombia-Purina Petcare \\
\hline 16 & Laboratorios Wyeth Inc. \\
\hline 17 & Susalud S.A. \\
\hline 18 & Nestlé de Colombia S.A. \\
\hline 19 & Branch of Microsoft Colombia Inc. \\
\hline 20 & Proexport Colombia \\
\hline 21 & Salud Total S.A. E.P.S. A.R.S. \\
\hline 22 & Hewlett Packard Colombia Ltda. \\
\hline 23 & Astrazeneca Colombia S.A. \\
\hline 24 & Oracle Colombia Ltda. \\
\hline 25 & Edatel S.A. E.S.P. \\
\hline 25 & Frytolay Colombia Ltda. \\
\hline 25 & Unidad prestadora de servicios de salud \\
\hline
\end{tabular}




\section{Bibliografía}

Blanco Valbuena Carlos ( 2008). El conocimiento y su gestión en la nueva economía. Revista Documentos de Adminstracion $\mathrm{N}^{\circ} 8$, enero 2008, Departamento de Administración de Empresas, Pontificia Universidad Javeriana.

Castellanos, Juan Gonzalo (2003). PyMEs innovadoras, cambio de estrategias e instrumentos. Revista EAN, No. 47.

Davenpñort T., PRUZAK L. (2001) Conocimiento en acción. Prentice Hall, Buenos Aires.

Goleman, Daniel(1998), La inteligencia emocional en la empresa Vergara, Argentina. http//: www.wyeth .com $\mathrm{http} / /:$ www.great place to work.com

Mendoza, José María. (1996). Aprendizaje organizacional para una época de cambios. Universidad del Norte.

NONAKA, Ikujiro. (2000). La Empresa creadora de conocimiento. Harvard Business Review. Gestión del Conocimiento. Editorial Deusto.

Ordoñez de Pablos P. (2003): Gestión del conocimiento y capital intelectual: Estudio de casos, En Ventura, J. y Ordoñez de Pablos P. (Coord.): Capital intelectual y aprendizaje organizativo: Nuevos desafíos para la empresa. Capítulo 5, pp.131-153

Pérez U.Rafael (2004). Liderazgo visionario. Revista EAN, No. 58, Bogotá, Septiembre Diciembre, 2006. Págs.79-85

Velásquez C., Andrés T. (2006). Diseño de organizaciones para la creación de conocimiento. Revista EAN. Na58. 